International Mathematical Forum, Vol. 8, 2013, no. 22, 1079 - 1090

HIKARI Ltd, www.m-hikari.com

http://dx.doi.org/10.12988/imf.2013.3482

\title{
On the Symplectomorphism Group of Product of Complex Projective Spaces ${ }^{1}$
}

\author{
Jiaji Ma and Guangcun $\mathrm{Lu}^{2}$
}

School of Mathematical Sciences, Beijing Normal University

Laboratory of Mathematics and Complex Systems

Ministry of Education, Beijing 100875, P.R. China

majiaji@mail.bnu.edu.cn, gclu@bnu.edu.cn

Copyright (c) 2013 Jiaji Ma and Guangcun Lu. This is an open access article distributed under the Creative Commons Attribution License, which permits unrestricted use, distribution, and reproduction in any medium, provided the original work is properly cited.

\begin{abstract}
Seidel proved in [1] that the homomorphism $\beta_{k}: \pi_{k}(\operatorname{Symp}(M, \omega)) \rightarrow$ $\pi_{k}(\operatorname{Diff}(M))$ induced by the inclusion is not surjective for $(M, \omega)=$ $\left(\mathbb{C} P^{N_{1}} \times \mathbb{C} P^{N_{2}}, \omega_{N_{1}} \times \omega_{N_{2}}\right)$ and each odd $k \leq \max \left\{2 N_{1}-1,2 N_{2}-1\right\}$, where $\omega_{n}$ is the standard symplectic form on $\mathbb{C} P^{n}$ with $\int_{\mathbb{C} P^{1}} \omega_{n}=1$. In this note, this profound result is generalized to the case $(M, \omega)=$ $\left(\mathbb{C} P^{N_{1}} \times \cdots \times \mathbb{C} P^{N_{m}}, \omega_{N_{1}} \times \cdots \times \omega_{N_{m}}\right)$ with arbitrary $m \geq 2$.
\end{abstract}

Mathematics Subject Classification: 53C15, 53D35, 37J05

Keywords: Complex projective space, symplectomorphism group, diffeomorphism group, parameterized Gromov-Witten invariants

\section{Introduction}

Let $\omega_{n}$ be the standard symplectic form on $\mathbb{C} P^{n}$, and let $\left(P_{m n}, \eta_{m n}\right)=\left(\mathbb{C} P^{m} \times\right.$ $\left.\mathbb{C} P^{n}, \omega_{m} \times \omega_{n}\right)$. For the homomorphisms $\beta_{k}: \pi_{k}\left(\operatorname{Symp}\left(P_{m n}, \eta_{m n}\right)\right) \rightarrow \pi_{k}\left(\operatorname{Diff}\left(P_{m n}\right)\right)$ induced by inclusion $\operatorname{Symp}\left(P_{m n}, \eta_{m n}\right) \hookrightarrow \operatorname{Diff}\left(P_{m n}\right)$, using the parameterized Gromov-Witten invariants (cf.[2]) Seidel [1] proved the following estimate:

$$
\begin{aligned}
\operatorname{rank}\left(\operatorname{coker} \beta_{k}\right) & \geq b_{2 m+1-k}\left(P_{m n}\right)-b_{2 m+1-k}\left(\mathbb{C} P^{m}\right)+ \\
& +b_{2 n+1-k}\left(P_{m n}\right)-b_{2 n+1-k}\left(\mathbb{C} P^{n}\right)>0,
\end{aligned}
$$

\footnotetext{
${ }^{1}$ Partially supported by the NNSF 10971014 and 11271044 of China, and PCSIRT.

${ }^{2}$ Corresponding author
} 
for each odd $k \leq \max \{2 m-1,2 n-1\}$, where $b_{*}$ are the Betti numbers. In particular, $\beta_{k}$ is not surjective. This is the first example of symplectic manifolds in which the homeomorphism $\beta_{k}: \pi_{k}(\operatorname{Symp}(M, \omega)) \rightarrow \pi_{k}(\operatorname{Diff}(M))$ is not surjective for dimension greater than 4 . The case that $m=n=k=1$ can be derived from a result of Gromov [3, 0.3.C]. This note shows that Seidel's method can still deal with symplectic manifolds $(M, \omega)=\left(\mathbb{C} P^{N_{1}} \times \cdots \times\right.$ $\left.\mathbb{C} P^{N_{m}}, \omega_{N_{1}} \times \cdots \times \omega_{N_{m}}\right)$ for each $m \geq 2$. The main result, Theorem 3.2, gives the estimate of $\operatorname{rank}\left(\operatorname{coker} \beta_{d-1}\right)$ via the Betti numbers for every even $d \geq 2$. In particular, Corollary 3.4 claims $\operatorname{rank}\left(\operatorname{coker} \beta_{k}\right) \geq m C_{N+m-\frac{k+1}{2}}^{m-1}-m$ for each odd $k \in[1,2 N-1]$ if $N_{1}=\cdots=N_{m}=N$, and $\operatorname{rank}\left(\operatorname{coker} \beta_{1}\right) \geq$ $C_{N_{1}+m-1}^{m-1}+(m-1) \sum_{k=0}^{N_{1}} C_{N+m-k-1}^{m-1}-m$ if $N_{1}<N_{2}=\cdots=N_{m}=N$.

\section{Deformations of the $\mathbb{Q}$-algebra $H^{*}(M)$}

For reader's convenience we review some necessary definitions and properties in [1]. All algebras are finite-dimensional, commutative (in the graded sense), and have a unit.

Definition 2.1 Let $R$ be a graded $\mathbb{F}$-algebra over a field $\mathbb{F}$. A deformation of $R$ of dimension $d>0$ consists of: (i) a graded $\mathbb{F}$-algebra $\widetilde{R}$, (ii) an element $t \in \widetilde{R}^{d}$ with $t^{2}=0$, such that the sequence $0 \rightarrow \widetilde{R} / t \widetilde{R} \rightarrow \widetilde{R} \rightarrow \widetilde{R} / t \widetilde{R} \rightarrow 0$ is exact, where the second arrow is $\times t$, (iii) a homomorphism of graded algebras $j: \widetilde{R} \rightarrow R$ which is surjective with kernel $t \widetilde{R}$.

One can also define the notion of isomorphisms between $d$-dimensional deformations of $R$. Denote by $\operatorname{Def}_{d}(R)$ the set of isomorphism classes of all $d$ dimensional deformations of $R$. It has a natural structure of an $\mathbb{F}$-vector space. In Remark 2.2 of [1], the author also gave the following equivalent definition of $\operatorname{Def}_{d}(R)$.

Definition 2.2 If $Z_{d}(R)$ denotes the space of $\mathbb{F}$-bilinear maps $\psi: R \times R \rightarrow R$ of degree $-d$ which are graded symmetry and satisfy

$$
(-1)^{d \operatorname{deg}(x)} x \psi(y, z)-\psi(x y, z)+\psi(x, y z)-\psi(x, y) z=0,
$$

and $B_{d}(R) \subset Z_{d}(R)$ is the subset of maps of the form

$$
\psi(x, y)=(-1)^{d \operatorname{deg}(x)} x \xi(y)-\xi(x y)+\xi(x) y,
$$

where $\xi: R \rightarrow R$ is some linear map of degree $-d$, then $\operatorname{Def}_{d}(R)=Z_{d}(R) / B_{d}(R)$.

So if odd dimension parts of $R$ are 0 , then $\operatorname{Def}_{d}(R)=0$ for all odd $d$.

Definition 2.3([1, Sec.6]) Let $R_{1}$ and $R_{2}$ be two graded $\mathbb{F}$-algebras and let $\widetilde{R}_{1}$ and $\widetilde{R}_{2}$ be their deformations of the same dimension, respectively. For the graded tensor product of $R_{1}$ and $R_{2}, R=R_{1} \otimes R_{2}$, define a deformation 
$(\widetilde{R}, t, j)$ of $R$ by $\widetilde{R}:=\widetilde{R}_{1} \bigotimes_{F[\epsilon] / \epsilon^{2}} \widetilde{R}_{2}$, where $\epsilon$ acts on $\widetilde{R}_{i}$ by multiplication with $t_{i}, t:=t_{1} \otimes 1=1 \otimes t_{2}$ and $j:=j_{1} \otimes j_{2}$. The triple $(\widetilde{R}, t, j)$ is called the exterior product of $\widetilde{R}_{1}$ and $\widetilde{R}_{2}$.

This notion can be generalized to the case of finitely many deformations of the same dimension.

Definition 2.4([1, Sec.6]) A deformation of $R$ is called split if it is isomorphic to an exterior product of finitely many deformations of the same dimension.

Denote by $\operatorname{Def}_{d}^{s}(R)$ the set of all spit deformations of $R$.

Definition 2.5 (semi-split) Following the notations in Definition 2.3, we say a deformation $(\widetilde{R}, t, j)$ of $R$ to be semi-split with respect to $R_{s}(s=1$ or 2$)$ if there is a deformation $\left(\widetilde{R}_{s}, t_{s}, j_{s}\right)$ of $R_{s}$ and a morphism $\tilde{f}_{s}: \widetilde{R}_{s} \rightarrow \widetilde{R}$ which lie over the natural inclusion $f_{s}: R_{s} \rightarrow \widetilde{R}$.

Theorem 2.6([1, Lemma 2.3]) Let $M$ be a compact smooth manifold, and let $\pi: E \rightarrow S^{d}$ be a smooth fibre bundle with fibre $M$. For some fixed $b_{0} \in S^{d}$ let $i: M \rightarrow E_{b_{0}}$ be a diffeomorphism. Let $\epsilon$ denote the generator of $H^{d}\left(S^{d}\right)$ and $t:=\pi^{*}(\epsilon)$. If $i^{*}$ is onto, then $\left(H^{*}(E ; \mathbb{F}), t, i^{*}\right)$ is a d-dimensional deformation of $H^{*}(M ; \mathbb{F})$.

According to [page 138,4], if $i^{*}$ is onto then $M$ is said to be totally nonhomologous to zero in $E$ with respect to the field $\mathbb{F}$.

Lemma 2.7 Let $\pi: E \rightarrow S^{d}$ be as in Theorem 2.6. Suppose that $d>0$ is even and that the cohomology $H^{*}(M ; \mathbb{F})$ has no nontrivial element of odd degree. Then $i^{*}$ is onto.

Proof. This easily follows from spectral sequences. In fact, the second page of the spectral sequence is zero except $(2 n, d)$ position. Then all elements are not changed under the differential $d_{r}$ for $r \neq d+1$. But on the $(d+1)$-th page, the differential $d_{d+1}=0$ since the cohomology lies in even degree. As in [Theorem 5.8,4] we may express $i^{*}$ by $d_{r}$, and deduce that $i^{*}$ is onto.

In the following we always assume $M=\mathbb{C} P^{N_{1}} \times \cdots \times \mathbb{C} P^{N_{m}}$ with $m \geq 2$. Then for each even $d>0$ and a smooth fibre bundle $\pi: E \rightarrow S^{d}$ with fibre $M$ Theorem 2.6 and Lemma 2.7 show that $\left(H^{*}(E ; \mathbb{Q}), t, i^{*}\right)$ is a $d$-dimensional deformation of

$$
R:=H^{*}\left(\mathbb{C} P^{N_{1}} \times \cdots \times \mathbb{C} P^{N_{m}} ; \mathbb{Q}\right)=H^{*}\left(\mathbb{C} P^{N_{1}} ; \mathbb{Q}\right) \otimes \cdots \otimes H^{*}\left(\mathbb{C} P^{N_{m}} ; \mathbb{Q}\right) .
$$

With the similar arguments to Sections 2, 3 in [1], we may get the following theorem, which classifies deformations of $R$.

Theorem 2.8 For each even $d \leq 2 \max _{s}\left\{N_{s}+1\right\}, \operatorname{Def}_{d}(R)$ is isomorphic to

$$
R^{2 N_{1}+2-d} \bigoplus \cdots \bigoplus R^{2 N_{m}+2-d}
$$

if $d>2$ (where $R^{2 N_{s}+2-d}=0$ if $d>2 N_{s}+2$ ), and isomorphic to

$$
R^{2 N_{1}} / \mathbb{Q}\left(N_{1}+1\right) u^{N_{1}} \bigoplus \cdots \bigoplus R^{2 N_{m}} / \mathbb{Q}\left(N_{m}+1\right) u^{N_{m}}
$$


if $d=2$.

Proof. Step 1. Each group of elements $a_{s} \in R^{2 N_{s}+2-d}, s=1, \cdots, m$, determines a deformation of $R$. In fact, let $u_{s}$ be the generators of $H^{2}\left(\mathbb{C} P^{N_{s}} ; \mathbb{Q}\right)$, $s=1, \cdots, m$. Then $R=\mathbb{Q}\left[u_{1}, \cdots, u_{m}\right] /\left(u_{1}^{N_{1}+1}, \cdots, u_{m}^{N_{m}+1}\right)$. Since $d \leq 2 N_{s}+2$ for some $s$, for each such $s$ we may write $a_{s}$ as

$$
a_{s}=\sum_{i_{1}+\cdots+i_{m}=N_{s}+1-d / 2} b_{s, i_{1} \cdots i_{m}} u_{1}^{i_{1}} u_{2}^{i_{2}} \cdots u_{m}^{i_{m}},
$$

where $b_{s, i_{1} \cdots i_{m}} \in \mathbb{Q}$ and $i_{1}, \cdots, i_{m}$ are nonnegative integers. Denote by $\widetilde{R}_{a_{1}, \cdots, a_{m}}$ the $\mathbb{Q}$-algebra with generators $\tilde{u}_{1}, \cdots, \tilde{u}_{m}, t$ of degrees $2, \cdots, 2, d$, and relations

$$
\left.\begin{array}{l}
\tilde{u}_{s}^{N_{s}+1}+t \sum_{i_{1}+\cdots+i_{m}=N_{s}+1-d / 2} b_{s, i_{1} \cdots i_{m}} \tilde{u}_{1}^{i_{1}} \tilde{u}_{2}^{i_{2}} \cdots \tilde{u}_{m}^{i_{m}}=0 \\
\text { for each } s \text { with } 2 N_{s}+2 \geq d, \quad \text { and } t^{2}=0 .
\end{array}\right\}
$$

Define the homomorphism $j: \widetilde{R}_{a_{1}, \cdots, a_{m}} \rightarrow R$ by $j\left(\tilde{u}_{s}\right)=u_{s}, s=1, \cdots, m$ and $j(t)=0$. It is easy to check that $\left(\widetilde{R}_{a_{1}, \cdots, a_{m}}, j, t\right)$ is a deformation of $R$.

Step 2. Each deformation $(\widetilde{R}, \bar{j}, \bar{t})$ of $R$ is isomorphic to some $\left(\widetilde{R}_{a_{1}, \cdots, a_{m}}, j, t\right)$ as in Step 1. Let $\tilde{v}_{s}$ be the lifts of $u_{s}$ in $\widetilde{R}$, i.e. $\bar{j}\left(\tilde{v}_{s}\right)=u_{s}, s=1, \cdots, m$. Since $\bar{j}\left(\tilde{v}_{s}^{N_{s}+1}\right)=u_{s}^{N_{s}+1}=0$, by Definition 2.1(iii) we have some $\tilde{a}_{s} \in \widetilde{R}$ such that $\tilde{v}_{s}^{N_{s}+1}=-\bar{t} \tilde{a}_{s}$ for $s=1, \cdots, m$. Set $a_{s}:=\bar{j}\left(\tilde{a}_{s}\right), s=1, \cdots, m$. Then $a_{s} \in R^{2 N_{s}+2-d}$ (by comparing their degrees) and hence can be written as the form in (2.1) if $2 N_{s}+2 \geq d$. It follows from these and Definition 2.1(ii) that there exist the following relations as in (2.2) for each $s$ with $2 N_{s}+2 \geq d$,

$$
\tilde{v}_{s}^{N_{s}+1}+\bar{t} \sum_{i_{1}+\cdots+i_{m}=N_{s}+1-d / 2} b_{s, i_{1} \cdots i_{m}} \tilde{v}_{1}^{i_{1}} \cdots \tilde{v}_{m}^{i_{m}}=0 \quad \text { and } \quad \bar{t}^{2}=0 .
$$

Using these it is easy to construct a homomorphism $\tilde{f}$ from $\widetilde{R}_{a_{1}, \cdots, a_{m}}$ to $\widetilde{R}$ by sending $\tilde{u}_{s}$ to $\tilde{v}_{\tilde{s}}, s=1, \cdots, m$ and $t$ to $\bar{t}$. Actually, it is a morphism from the deformations $\left(\widetilde{R}_{a_{1}, \cdots, a_{m}}, j, t\right)$ to $(\widetilde{R}, \bar{j}, \bar{t})$ since $\tilde{f}(t)=\bar{t}$ and $\bar{j} \circ \tilde{f}=j$. By the claim below [Definition 2.1, 1] $(\widetilde{R}, \bar{j}, \bar{t})$ and $\left(\widetilde{R}_{a_{1}, \cdots, a_{m}}, j, t\right)$ are isomorphic.

Step 3. Let $\left(\widetilde{R}_{a_{1}, \cdots, a_{m}}, t, j\right)$ and $\left(\widetilde{R}_{a_{1}^{\prime}, \cdots, a_{m}^{\prime}}, t^{\prime}, j^{\prime}\right)$ be two deformations of $R$ as in Step 1, and let $\psi$ be an isomorphism from the former to the latter. We claim:

- If $d>2$, then $\widetilde{R}_{a_{1}, \cdots, a_{m}}$ is isomorphic to $\widetilde{R}_{a_{1}^{\prime}, \cdots, a_{m}^{\prime}}$ if and only if $\left(a_{1}, \cdots, a_{m}\right)=$ $\left(a_{1}^{\prime}, \cdots, a_{m}^{\prime}\right)$.

- If $d=2$, then $\widetilde{R}_{a_{1}, \cdots, a_{m}}$ is isomorphic to $\widetilde{R}_{a_{1}^{\prime}, \cdots, a_{m}^{\prime}}$ if and only if $a_{s}-a_{s}^{\prime}$ is a multiple of $\left(N_{s}+1\right) u_{s}^{N_{s}}, s=1, \cdots, m$.

Indeed, suppose that $\tilde{u}_{1}^{\prime}, \cdots, \tilde{u}_{m}^{\prime}, t^{\prime}$ are generators of $\widetilde{R}_{a_{1}^{\prime}, \cdots, a_{m}^{\prime}}$ of degrees $2, \cdots, 2, d$ as in Step 1 . Then $j\left(\tilde{u}_{s}\right)=j^{\prime}\left(\tilde{u}_{s}{ }^{\prime}\right)=u_{s}, s=1, \cdots, m$. Since $j=j^{\prime} \circ \psi$, we have $j^{\prime}\left(\psi\left(\tilde{u}_{s}\right)-\tilde{u}_{s}{ }^{\prime}\right)=0$ and hence $\psi\left(\tilde{u}_{s}\right)-\tilde{u}_{s}{ }^{\prime}=t^{\prime} \tilde{r}_{s}$ for some $\tilde{r}_{s} \in \widetilde{R}_{a_{1}^{\prime}, \cdots, a_{m}^{\prime}}$ by Definition 2.1(iii), $s=1, \cdots, m$. 
If $d>2$ we have $\tilde{r}_{s}=0$ and hence

$$
\psi\left(\tilde{u}_{s}\right)=\tilde{u}_{s}^{\prime}, \quad s=1, \cdots, m .
$$

For each $s$ with $2 N_{s}+2 \geq d$ we may write

$$
\begin{aligned}
\tilde{a}_{s} & =\sum_{i_{1}+\cdots+i_{m}=N_{s}+1-d / 2} b_{s, i_{1}, \ldots i_{m}} \tilde{u}_{1}^{i_{1}} \tilde{u}_{2}^{i_{2}} \ldots \tilde{u}_{m}^{i_{m}} \\
\tilde{a}_{s}^{\prime} & =\sum_{i_{1}+\cdots+i_{m}=N_{s}+1-d / 2} b_{s, i_{1}, \ldots i_{m}}^{\prime}\left(\tilde{u}_{1}^{\prime}\right)^{i_{1}}\left(\tilde{u}_{2}^{\prime}\right)^{i_{2}} \ldots\left(\tilde{u}_{m}^{\prime}\right)^{i_{m}} .
\end{aligned}
$$

By (2.2) we have $\left(\tilde{u}_{s}\right)^{N_{s}+1}+t \tilde{a}_{s}=0$ and $\left(\tilde{u}_{s}^{\prime}\right)^{N_{s}+1}+t^{\prime} \tilde{a}_{s}^{\prime}=0, s=1, \cdots, m$. Since $\psi(t)=t^{\prime}$, it follows from these and $(2.3)$ that $t^{\prime}\left(\psi\left(\tilde{a}_{s}\right)-\tilde{a}_{s}^{\prime}\right)=0$. By Definition 2.1(ii) this leads to $\psi\left(\tilde{a}_{s}\right)-\tilde{a}_{s}^{\prime}=0$. Moreover, since $a_{s}=j\left(\tilde{a}_{s}\right)$, $a_{s}^{\prime}=j^{\prime}\left(\tilde{a}_{s}^{\prime}\right), s=1, \cdots, m$, and $j^{\prime} \circ \psi=j$, we arrive at $a_{s}=j\left(\tilde{a}_{s}\right)=j^{\prime} \circ \psi\left(\tilde{a}_{s}\right)=$ $j^{\prime}\left(\tilde{a}_{s}^{\prime}\right)=a_{s}^{\prime}$ for $s=1, \cdots, m$ because $a_{s}=a_{s}^{\prime}=0$ for $s$ with $2 N_{s}+2<d$. The first claim is proved.

If $d=2$, we may assume $\psi\left(\tilde{u}_{s}\right)-\tilde{u}_{s}{ }^{\prime}=t^{\prime} \beta_{s}=\beta_{s} t^{\prime}$ for some $\beta_{s} \in \tilde{R}_{a_{1}^{\prime}, \ldots, a_{m}^{\prime}}^{0}$, $s=1, \cdots, m$. Since $\tilde{u}_{s}^{N_{s}+1}+t \tilde{a}_{s}=0$ and $\left(\tilde{u}_{s}^{\prime}\right)^{N_{s}+1}+t^{\prime} \tilde{a}_{s}^{\prime}=0$, we get

$$
\begin{aligned}
0 & =\psi\left(\tilde{u}_{s}^{N_{s}+1}+t \tilde{a}_{s}\right) \\
& =\left(\psi\left(\tilde{u}_{s}\right)\right)^{N_{s}+1}+\psi(t) \psi\left(\tilde{a}_{s}\right) \\
& =\left(\tilde{u}_{s}^{\prime}+\beta_{s} t^{\prime}\right)^{N_{s}+1}+\psi(t) \psi\left(\tilde{a}_{s}\right) \\
& =\left(\tilde{u}_{s}^{\prime}\right)^{n_{s}+1}+\left(N_{s}+1\right) \beta_{s} t^{\prime}\left(\tilde{u}_{s}^{\prime}\right)^{N_{s}}+\psi(t) \psi\left(\tilde{a}_{s}\right) \\
& =-t^{\prime} \tilde{a}_{s}^{\prime}+\left(N_{s}+1\right) \beta_{s} t^{\prime}\left(\tilde{u}_{s}^{\prime}\right)^{N_{s}}+\psi(t) \psi\left(\tilde{a}_{s}\right) \\
& =t^{\prime}\left(\psi\left(\tilde{a}_{s}\right)-\tilde{a}_{s}^{\prime}+\left(N_{s}+1\right) \beta_{s}\left(\tilde{u}_{s}^{\prime}\right)^{N_{s}}\right)
\end{aligned}
$$

because $\psi(t)=t^{\prime}, s=1, \cdots, m$. By the exact sequence in Definition 2.1(ii) we deduce that $\psi\left(\tilde{a}_{s}\right)-\tilde{a}_{s}^{\prime}+\left(N_{s}+1\right) \beta_{s}\left(\tilde{u}_{s}^{\prime}\right)^{N_{s}}=0$ and hence

$$
\begin{aligned}
& 0=j^{\prime}\left(\psi\left(\tilde{a}_{s}\right)-\tilde{a}_{s}^{\prime}+\left(N_{s}+1\right) \beta_{s}\left(\tilde{u}_{s}^{\prime}\right)^{N_{s}}\right) \\
& =j^{\prime} \circ \psi\left(\tilde{a}_{s}\right)-j^{\prime}\left(\tilde{a}_{s}^{\prime}\right)+\left(N_{s}+1\right) j^{\prime}\left(\beta_{s}\right)\left(j^{\prime}\left(\tilde{u}_{s}^{\prime}\right)\right)^{N_{s}} \\
& =a_{s}-a_{s}^{\prime}+\left(N_{s}+1\right) j^{\prime}\left(\beta_{s}\right)\left(u_{s}\right)^{N_{s}}
\end{aligned}
$$

for $s=1, \cdots, m$. Theorem 2.8 is proved.

Theorem 2.9([Prop.4.2, 1]) For a compact manifold $(M, \omega)$ suppose that there exists a spherical homology class $A \in H_{2}^{s}(M ; \mathbb{Z})$ such that $\omega(A)$ is positive and generates the period group $\omega\left(H_{2}^{s}(M ; \mathbb{Z})\right) \subset \mathbb{R}$. The classical method can define the Gromov-Witten invariant $\mathcal{G W}_{A, 0,3}^{(M, \omega)}: H^{*}(M ; \mathbb{Q})^{\otimes} \rightarrow \mathbb{Q}$, which can be written as a (graded) symmetric, bilinear map $\psi_{A}: H^{*}(M ; \mathbb{Q}) \times H^{*}(M ; \mathbb{Q}) \longrightarrow$ $H^{*}(M ; \mathbb{Q})$ of degree $-2 c_{1}(A)$. Let $(E, \pi, i, \Omega)$ be a symplectic fibre bundle over 
$S^{d}(d \geq 2)$ with fibre $(M, \omega)$, such that $i^{*}$ is onto. Then the deformation of $R:=H^{*}(M ; \mathbb{Q})$ determined by $H^{*}(E ; \mathbb{Q})$ admits an extension of $\psi_{A}$ in the sense of $[$ Def.4.1, 1].

The proof of this result was completed with the parametrized GromovWitten invariants. Using this theorem Seidel obtained:

Theorem 2.10([Prop.6.5, 1]) Suppose that $\left(M^{\prime}, \omega^{\prime}\right)$ is a compact manifold whose cohomology ring $H^{*}\left(M^{\prime} ; \mathbb{Q}\right)$ is generated by $H^{2}\left(M^{\prime} ; \mathbb{Q}\right)$, and that $\omega^{\prime}\left(H_{2}^{s}\left(M^{\prime} ; \mathbb{Z}\right)\right) \subset$ $\mathbb{Z}$. Let $(E, \pi, i, \Omega)$ be a symplectic fibre bundle with fibre $(M, \omega)=\left(M^{\prime}, \omega^{\prime}\right) \times$ $\left(\mathbb{C} P^{n}, \omega_{n}\right)$ over $S^{d}$ for some even $d>0$. Then the deformation of $R=$ $H^{*}(M ; \mathbb{Q})$ given by $H^{*}(E ; \mathbb{Q})$ is semi-split with respect to $H^{*}\left(M^{\prime} ; \mathbb{Q}\right)$.

Corresponding to split theorem [Prop.6.2, 1] we have

Theorem 2.11 Let $(E, \pi, i, \Omega)$ be a symplectic fibre bundle over $S^{d}$ with fibre $(M, \omega)=\left(\mathbb{C} P^{N_{1}} \times \cdots \times \mathbb{C} P^{N_{m}}, \omega_{N_{1}} \times \cdots \times \omega_{N_{m}}\right)$ for some even $d>0$. Then the deformation determined by $H^{*}(E ; \mathbb{Q})$ is split, i.e. it is the exterior product of certain deformations of $H^{*}\left(\mathbb{C} P^{N_{s}} ; \mathbb{Q}\right), s=1, \cdots, m$.

Proof. For each $s=1, \cdots, m$, we can write $(M, \omega)=\left(M_{s}^{\prime}, \omega_{s}^{\prime}\right) \times\left(\mathbb{C} P^{N_{s}}, \omega_{N_{s}}\right)$, where $\left(M_{s}^{\prime}, \omega_{s}^{\prime}\right)=\left(\mathbb{C} P^{N_{1}} \times \cdots \times \widehat{\mathbb{C} P^{N_{s}}} \times \cdots \times \mathbb{C} P^{N_{m}}, \omega_{N_{1}} \times \cdots \widehat{\omega_{N_{s}}} \times \cdots \times \omega_{N_{m}}\right)$. Clearly, $H^{2}\left(M_{s}^{\prime} ; \mathbb{Q}\right)$ generates the ring $H^{*}\left(M_{s}^{\prime} ; \mathbb{Q}\right)$, and $\omega_{s}^{\prime}\left(H_{2}^{s}\left(M_{s}^{\prime} ; \mathbb{Z}\right)\right) \subset \mathbb{Z}$. By Theorem 2.6 and Lemma 2.7 both $H^{*}(M ; \mathbb{Q})$ and $H^{*}\left(M_{s}^{\prime} ; \mathbb{Q}\right)$ have $d$-dimension deformations. In particular, $\left(H^{*}(E ; \mathbb{Q}), t, j=i^{*}\right)$ is a $d$-dimension deformation of $H^{*}(M ; \mathbb{Q})$. Now Theorem 2.10 tells us that $H^{*}(E ; \mathbb{Q})$ is semi-split with respect to $R_{s}:=H^{*}\left(M_{s}^{\prime} ; \mathbb{Q}\right)$. Hence there exists a deformation $\left(\widetilde{R}_{s}, t_{s}, j_{s}\right)$ and a morphism $\tilde{f}_{s}: \widetilde{R}_{s} \rightarrow H^{*}(E ; \mathbb{Q})$ covering the inclusion $f_{s}: R_{s} \rightarrow H^{*}(M ; \mathbb{Q})$.

Suppose that $\tilde{f}_{s}\left(\tilde{r}_{s}\right)=0$. Since $f_{s}$ is injective and $j \tilde{f}_{s}=f_{s} j_{s}$, we have $j_{s}\left(\tilde{r}_{s}\right)=0$. It follows from Definition 2.1(iii) that $\tilde{r}_{s}=t_{s} \tilde{r}_{s}^{\prime}$ for some $\tilde{r}_{s}^{\prime} \in \widetilde{R}_{s}$. Note that $t \tilde{f}_{s}\left(\tilde{r}_{s}^{\prime}\right)=\tilde{f}_{s}\left(t_{s}\right) \tilde{f}_{s}\left(\tilde{r}_{s}^{\prime}\right)=\tilde{f}_{s}\left(t_{s} \tilde{r}_{s}^{\prime}\right)=\tilde{f}_{s}\left(\tilde{r}_{s}\right)=0$. By the exact sequence in Definition 2.1(ii) we deduce that $\tilde{f}_{s}\left(\tilde{r}_{s}^{\prime}\right)=0$. This implies that $\tilde{r}_{s}^{\prime}=t_{s} \tilde{\gamma}_{s}$ for some $\tilde{\gamma}_{s} \in \widetilde{R}_{s}$ again. Hence $\tilde{r}_{s}=t_{s} \tilde{r}_{s}^{\prime}=\left(t_{s}\right)^{2} \tilde{\gamma}_{s}=0$, and $\tilde{f}_{s}$ is injective.

Identifying $\widetilde{R}_{s}$ and its image via $\tilde{f}_{s}$ and checking the proof of Proposition 6.5 in [1], the deformation can be chosen good enough, for example, we can require that $\widetilde{R}_{s}$ is generated by $t, \tilde{u}_{1}, \cdots \widehat{\tilde{u}}_{s}, \cdots, \tilde{u}_{m}$. Then $W_{s}:=\bigcap_{j \neq s} \widetilde{R}_{j}=$ $\operatorname{span}\left\{t, \tilde{u}_{s}\right\}$ is a $d$-dimension deformation of $H^{*}\left(\mathbb{C} P^{N_{s}} ; \mathbb{Q}\right)$ which covers the inclusion $H^{*}\left(\mathbb{C} P^{N_{s}} ; \mathbb{Q}\right) \rightarrow H^{*}(E ; \mathbb{Q})$. It follows that $H^{*}(E ; \mathbb{Q})$ is the wedge product of $W_{s}, s=1, \cdots, m$.

From the proof of this theorem one may see that the conditions $\int_{\mathbb{C} P^{1}} \omega_{N_{s}}=$ $1, s=1, \cdots, m$, are necessary for us using Theorem 2.10. 


\section{$3 \quad$ Results and proofs}

Denote by $\mathcal{F}\left(S^{d}, M\right)$ the set of all isomorphism classes of smooth fibre bundles over $S^{d}$ with fibre $M$. It is a group under the operation of fibre connected sum, and is isomorphic to $\pi_{d-1}(\operatorname{Diff}(M))$. Fix a base point $b_{0} \in S^{d}$. We have an explicit isomorphism obtained by the clutching construction,

$$
\mathcal{I}_{d}: \pi_{d-1}(\operatorname{Diff}(M)) \rightarrow \mathcal{F}\left(S^{d}, M\right),[\phi] \mapsto\left[E_{\phi}, \pi_{\phi}, i_{\phi}\right]
$$

where $\phi:\left(S^{d-1}, b_{0}\right) \rightarrow(\operatorname{Diff}(M), i d)$ and $i_{\phi}: M \rightarrow\left(E_{\phi}\right)_{b_{0}}$ is a diffeomorphism. Consider the subgroup $G_{d-1}=\left\{[\phi] \in \pi_{d-1}(\operatorname{Diff}(M)) \mid i_{\phi}^{*}\right.$ is onto $\}$. By Lemma 2.7 it is equal to $\pi_{d-1}(\operatorname{Diff}(M))$ provided that $d>0$ is even and that $H^{*}(M ; \mathbb{F})$ has no nontrivial element of odd degree. Theorem 2.6 defines a homeomorphism

$$
\alpha_{d-1}: G_{d-1} \rightarrow \operatorname{Def}_{d}\left(H^{*}(M ; \mathbb{Q})\right) .
$$

Corresponding to [Prop.3.1, 1] we have:

Theorem 3.1 For an even $0<d \leq 2 \max _{s}\left\{N_{s}+1\right\}$ and $(M, \omega)=\left(\mathbb{C} P^{N_{1}} \times\right.$ $\left.\cdots \times \mathbb{C} P^{N_{m}}, \omega_{N_{1}} \times \cdots \times \omega_{N_{m}}\right)$, the homomorphism

$$
\alpha_{d-1} \bigotimes i d_{\mathbb{Q}}: \pi_{d-1}(\operatorname{Diff}(M)) \otimes \mathbb{Q}=G_{d-1} \otimes \mathbb{Q} \rightarrow \operatorname{Def}_{d}(R)
$$

is surjective.

Proof. We only need to prove that each $0 \oplus 0 \cdots \oplus R^{2 N_{j}+2-d} \oplus 0 \cdots \oplus 0$ sits in the image of $\alpha_{d-1} \otimes i d_{\mathbb{Q}}$ for each $j$ with $2 N_{j}+2>d$. Since the proofs are similar, without loss of generality we may assume $j=1$. We also write $p=d / 2$ for conveniences. By Theorem 2.8

$$
R^{2 N_{1}+2-d}=\sum_{\substack{k_{1}+\cdots+k_{m} \\ \& k_{s} \leq N_{s} \forall s}} \mathbb{Q} u_{1}^{k_{1}} u_{2}^{k_{2}} \cdots u_{m}^{k_{m}} .
$$

Fix a group of nonnegative integers $i_{1}, \cdots, i_{m}$ such that $i_{1}+\cdots+i_{m}=N_{1}+1-p$ and $i_{s} \leq N_{s} \forall s \leq m$. It suffices to prove

Claim $\mathbf{A}$ The deformation $\widetilde{R}_{a 0 \cdots 0}$ associated with element

$$
a=u_{1}^{i_{1}} u_{2}^{i_{2}} \cdots u_{m}^{i_{m}}
$$

is contained in the image of $\alpha_{d-1} \otimes i d_{\mathbb{Q}}$.

Since $i_{2}+p \in\left[p, N_{2}+p\right]$, by [Lemma 3.2, 1] we can find a complex vector bundle of rank $i_{2}+p, \eta_{i_{2}} \rightarrow \mathbb{C} P^{N_{2}} \times S^{d}$, such that $\eta_{i_{2}} \mid \mathbb{C} P^{N_{2}} \times b_{0}$ is trivial and that $c_{s}\left(\eta_{i_{2}}\right) \neq 0$ with $s=i_{2}+p$. Clearly, $c_{s}\left(\eta_{i_{2}}\right)=0$ for $1 \leq s<p$ (if $p>1$ ). Moreover, the first condition implies that the Chern classes of it can be written as

$$
c_{s}\left(\eta_{i_{2}}\right)=\gamma_{s}\left(u_{2}^{s-p}\right) \otimes \epsilon, \quad \gamma_{s} \in \mathbb{Z}, \forall s \geq p
$$


where $u_{2}$ and $\epsilon$ are the generators of $H^{2}\left(\mathbb{C} P^{N_{2}} ; \mathbb{Q}\right)$ and $H^{d}\left(S^{d} ; \mathbb{Q}\right)$, respectively.

Pull $\eta_{i_{2}}$ back to $\mathbb{C} P^{N_{2}} \times \cdots \times \mathbb{C} P^{N_{m}} \times S^{d}$ via the obvious projection to $\mathbb{C} P^{N_{2}} \times S^{d}$, and denote it by $\widetilde{\eta}_{i_{2}}$. Then we have also

$$
\left.\begin{array}{l}
c_{s}\left(\widetilde{\eta}_{i_{2}}\right)=0 \quad \forall 0<s<p(\text { if } p>1) \\
c_{s}\left(\widetilde{\eta}_{i_{2}}\right)=\gamma_{s}\left(u_{2}^{s-p}\right) \otimes \epsilon, \quad \gamma_{s} \in \mathbb{Z}, \forall s \geq p
\end{array}\right\}
$$

For a $s \in\{3, \cdots, m\}$, if $i_{s} \geq 1$ we may use the obstruction theory to choose a complex line bundle $L_{s} \rightarrow \mathbb{C} P^{N_{s}}$ such that $c_{1}\left(L_{s}\right)=u_{s}$, and then set $\xi_{i_{s}}$ to be the sum of $i_{s}$ line bundles $L_{s}$. We get that $c_{j}\left(\xi_{i_{s}}\right)=\tau_{j} u_{s}^{j}$ with $\tau_{j} \in \mathbb{Z} \forall j$, and specially

$$
c_{i_{s}}\left(\xi_{i_{s}}\right)=u_{s}^{i_{s}}
$$

Pull $\xi_{i_{s}}$ back to $\mathbb{C} P^{N_{2}} \times \cdots \times \mathbb{C} P^{N_{m}} \times S^{d}$, and denote it by $\widetilde{\xi}_{i_{s}}$. Let $\varepsilon$ denote the trivial complex line bundle over $\mathbb{C} P^{N_{2}} \times \cdots \times \mathbb{C} P^{N_{m}} \times S^{d}$. Define

$$
\zeta:= \begin{cases}\bigoplus_{s=3, i_{s}>0}^{m} \widetilde{\xi}_{i_{s}} \bigoplus \widetilde{\eta}_{i_{2}} & \text { if } i_{1}=0 \\ \widetilde{\xi}_{s=3, i_{s}>0}^{m} \widetilde{\xi}_{i_{s}} \bigoplus \widetilde{\eta}_{i_{2}} \bigoplus \varepsilon^{i_{1}} & \text { if } i_{1}>0\end{cases}
$$

and $i:=\sum_{s=2}^{m} i_{s}+p$. Then $\zeta$ is a complex vector bundle over $\mathbb{C} P^{N_{2}} \times \cdots \times$ $\mathbb{C} P^{N_{m}} \times S^{d}$ of rank $\sum_{s=1}^{m} i_{s}+p=N_{1}+1$ with the Chern classes

$$
c_{i}(\zeta)=\gamma_{i_{2}+p} u_{2}^{i_{2}} u_{3}^{i_{3}} \cdots u_{m}^{i_{m}} \otimes \epsilon, \quad c_{0}(\zeta)=1 \quad \text { and } \quad c_{j}(\zeta)=0 \text { for other } j
$$

by (3.2) and (3.3), where $\gamma_{i_{2}+p}$ is as in (3.2). Note that we can always write

$$
c_{s}(\zeta)=\sum_{\substack{j_{2}+\cdots+j_{m}=s \\ \& j_{2} \geq p}} \gamma_{s, j_{2} \cdots j_{m}} u_{2}^{j_{2}-p} u_{3}^{j_{3}} \cdots u_{m}^{j_{m}} \otimes \epsilon
$$

with $\gamma_{s, j_{2} \cdots j_{m}} \in \mathbb{Z}$ for $s=1, \cdots, i$.

Let $\pi: \mathbb{P}(\zeta) \rightarrow \mathbb{C} P^{N_{2}} \times \cdots \times \mathbb{C} P^{N_{m}} \times S^{d}$ be the projectivized bundle of the bundle $\zeta$. By $\mathbb{P}(\zeta) \rightarrow \mathbb{C} P^{N_{2}} \times \cdots \times \mathbb{C} P^{N_{m}} \times S^{d} \rightarrow S^{d}$ we may view $\mathbb{P}(\zeta)$ as a bundle over $S^{d}$ with fiber $\mathbb{C} P^{N_{1}} \times \cdots \times \mathbb{C} P^{N_{m}}$. So the deformation of $R$ determined by $\mathbb{P}(\zeta)$ as in Theorem 2.6 sits in the image of $\alpha_{d-1} \otimes i d_{\mathbb{Q}}$. From the Leary-Hirsch theorem we know that $H^{*}(\mathbb{P}(\zeta) ; \mathbb{Q})$ is a free module over $H^{*}\left(\mathbb{C} P^{N_{2}} \times \cdots \times \mathbb{C} P^{N_{m}} \times S^{d} ; \mathbb{Q}\right)$ with base $\left\{1, v, \cdots, v^{N_{1}}\right\}$. Here the class $v \in H^{2}(\mathbb{P}(\zeta) ; \mathbb{Q})$ satisfies a relation

$$
v^{N_{1}+1}+\sum_{s=1}^{N_{1}+1} \pi^{*} c_{s}(\zeta) v^{N_{1}+1-s}=v^{N_{1}+1}+\sum_{s=p}^{i} \pi^{*} c_{s}(\zeta) v^{N_{1}+1-s}=0
$$


(Actually, $v$ restricts to a generator of $H^{2}\left(\mathbb{P}(\zeta)_{x} ; \mathbb{Q}\right)$ on the fiber $\mathbb{P}(\zeta)_{x} \equiv \mathbb{C} P^{N_{1}}$ at $\left.x \in \mathbb{C} P^{N_{2}} \times \cdots \times \mathbb{C} P^{N_{m}} \times S^{d}\right)$. Take $\tilde{u}_{1}=v, \tilde{u}_{s}=\pi^{*} u_{s}, s=2, \cdots, m$, and $t=\pi^{*} \epsilon$. It follows from these, (3.4) and (3.5) that

$$
\tilde{u}_{1}^{N_{1}+1}+t \sum_{s=p}^{i} \sum_{\substack{j_{2}+\cdots+j_{m}=s \\ \& j_{2} \geq p}} \gamma_{s, j_{2} \cdots j_{m}} \tilde{u}_{1}^{N_{1}+1-s} \tilde{u}_{2}^{j_{2}-p} \tilde{u}_{3}^{j_{3}} \cdots \tilde{u}_{m}^{j_{m}}=0 .
$$

This shows that the deformation of $R$ determined by $\mathbb{P}(\zeta)$ as in Theorem 2.6 is isomorphic $\widetilde{R}_{b, 0, \ldots 0}$, where

$$
b=\sum_{s=p}^{i} \sum_{\substack{j_{2}+\cdots+j_{m}=s \\ \& j_{2} \geq p}} \gamma_{s, j_{2} \cdots j_{m}} u_{1}^{N_{1}+1-s} u_{2}^{j_{2}-p} u_{3}^{j_{3}} \cdots u_{m}^{j_{m}}
$$

by (3.4). Hence $\widetilde{R}_{b, 0, \ldots 0}$ is in the image of $\alpha_{d-1} \otimes i d_{\mathbb{Q}}$.

Let us write $b=b_{p}+b_{p+1}+\cdots+b_{i}$, where

$$
\begin{aligned}
b_{p}= & \gamma_{p, p 0 \cdots 0} u_{1}^{N_{1}+1-p}, \\
b_{p+1}= & \sum_{\substack{j_{2}+\cdots+j_{m}=p+1 \\
\& j_{2} \geq p}} \gamma_{p+1, j_{2} \cdots j_{m}} u_{1}^{N_{1}-p} u_{2}^{j_{2}-p} u_{3}^{j_{3}} \cdots u_{m}^{j_{m}}+b_{p}, \\
b_{i-1}= & \sum_{\substack{j_{2}+\cdots+j_{m}=i-1 \\
\& j_{2} \geq p}} \gamma_{i-1, j_{2} \cdots j_{m}} u_{1}^{N_{1}+2-i} u_{2}^{j_{2}-p} u_{3}^{j_{3}} \cdots u_{m}^{j_{m}}+b_{i-2}, \\
b= & \gamma_{i_{2}+p} u_{1}^{N_{1}+1-i} u_{2}^{i_{2}} u_{3}^{i_{3}} \cdots u_{m}^{i_{m}}+b_{i-1} .
\end{aligned}
$$

We shall prove that $\widetilde{R}_{b_{k}, 0, \ldots 0}, k=p, \cdots, i-1$ (if $i>p$ ), are in in the image of $\alpha_{d-1} \otimes i d_{\mathbb{Q}}$, which lead to Claim A.

Step 1. $\widetilde{R}_{a, 0, \ldots 0}$ is in the image of $\alpha_{d-1} \otimes i d_{\mathbb{Q}}$ with $a=u_{1}^{N_{1}+1-p}$.

Let $\widetilde{\eta}_{0}$ be obtained by taking $i_{2}=0$ in $(3.2)$. Then $c_{s}\left(\widetilde{\eta}_{0}\right)=0$ for $s \neq p$, and $c_{p}\left(\widetilde{\eta}_{0}\right)=\gamma_{p} \epsilon$ with $\gamma_{p} \in \mathbb{Z} \backslash\{0\}$. Set $\zeta=\widetilde{\eta}_{0} \oplus \varepsilon^{N_{1}+1-p}$. It is a complex vector bundle of rank $N_{1}+1$, and $c_{s}(\zeta)=c_{s}\left(\widetilde{\eta}_{0}\right) \forall s$. As above we have $u_{1}^{N_{1}+1}+c_{p}(\zeta) u_{1}^{N_{1}+1-p}=0$. So the deformation of $R$ determined by $\mathbb{P}(\zeta)$ is $\widetilde{R}_{a_{p}, 0, \ldots 0}$, where $a_{p}=\gamma_{p} u_{1}^{N_{1}+1-p}$. The desired conclusion follows immediately.

If $i>p$ we proceed with the following:

Step 2. Given nonnegative integers $j_{2}, \cdots, j_{m}$ satisfying $j_{2}+\cdots+j_{m}=p+1$ and $j_{2} \geq p, \widetilde{R}_{a, 0, \ldots 0}$ is in the image of $\alpha_{d-1} \otimes i d_{\mathbb{Q}}$ with $a=u_{1}^{N_{1}-p} u_{2}^{j_{2}-p} u_{3}^{j_{3}} \cdots u_{m}^{j_{m}}$.

As in the above construction of $b$ in (3.6) we may obtain an element

$$
a_{p+1}=\alpha_{p+1, j_{2} \cdots j_{m}} u_{1}^{N_{1}-p} u_{2}^{j_{2}-p} u_{3}^{j_{3}} \cdots u_{m}^{j_{m}}+\alpha_{p} u_{1}^{N_{1}+1-p}
$$


with $\alpha_{p+1, j_{2} \cdots j_{m}} \in \mathbb{Z}$ and $\alpha_{p} \in \mathbb{Z}$, such that $\widetilde{R}_{a_{p+1}, 0, \ldots 0}$ is in the image of $\alpha_{d-1} \otimes i d_{\mathbb{Q}}$. This and Step 1 give rise to the expected result. In particular we deduce that $\widetilde{R}_{b_{p+1}, 0, \ldots 0}$ is in the image of $\alpha_{d-1} \otimes i d_{\mathbb{Q}}$.

If $i>p+1$, we proceed with the next step. Finally, we obtain that $\widetilde{R}_{a, 0, \ldots 0}$ is in the image of $\alpha_{d-1} \otimes i d_{\mathbb{Q}}$ with $a=u_{1}^{N_{1}+1-i} u_{2}^{i_{2}} u_{3}^{i_{3}} \cdots u_{m}^{i_{m}}$. Claim A is proved.

With the same arguments as in [Section 7, 1] Theorem 2.11 and Theorem 3.1 may lead to the following (3.7) and (3.8), respectively.

Theorem 3.2 For $(M, \omega)=\mathbb{C} P^{N_{1}} \times \cdots \times \mathbb{C} P^{N_{m}}$ and any positive even $d \leq \max \left\{2 N_{1}, \cdots, 2 N_{m}\right\}$ let $\beta_{d-1}: \pi_{d-1}(\operatorname{Symp}(M, \omega)) \rightarrow \pi_{d-1}(\operatorname{Diff}(M))$ be the inclusion, and let $i$ denote the natural inclusion $\operatorname{Def}_{d}^{s}(R) \hookrightarrow \operatorname{Def}_{d}(R)$, where $R:=H^{*}(M ; \mathbb{Q})$. Then

$$
\begin{aligned}
& \left(\alpha_{d-1} \otimes i d_{\mathbb{Q}}\right) \circ\left(\beta_{d-1} \otimes i d_{\mathbb{Q}}\right)=i \circ\left(\left.\alpha_{d-1} \otimes i d_{\mathbb{Q}}\right|_{\pi_{d-1}(\operatorname{Symp}(M, \omega)) \otimes \mathbb{Q}}\right), \\
& \operatorname{rank}\left(\operatorname{coker} \beta_{d-1}\right) \geq \operatorname{dim}_{\operatorname{Def}_{d}}(R)-\operatorname{dim}_{\operatorname{Def}_{d}^{s}}(R)
\end{aligned}
$$

In particular, for $d>2, \operatorname{Def}_{d}^{s}(R)=\bigoplus_{i=1}^{m} H^{2 N_{i}+2-d}\left(\mathbb{C} P^{N_{i}}\right)$ and hence

$$
\operatorname{rank}\left(\operatorname{coker} \beta_{d-1}\right) \geq \sum_{i=1}^{m} b_{2 N_{i}+2-d}(M)-\sum_{i=1}^{m} b_{2 N_{i}+2-d}\left(\mathbb{C} P^{2 N_{i}}\right) ;
$$

for $d=2, \operatorname{Def}_{2}^{s}(R)=0$ and so

$$
\operatorname{rank}\left(\operatorname{coker} \beta_{1}\right) \geq \operatorname{dim} \operatorname{Def}_{2}(R)=\sum_{i=1}^{m} \operatorname{dim} R^{2 N_{i}}-m
$$

By (3.9), (3.10) and Kunneth formula, we know that $\beta_{d-1}$ is not surjective for any even $d \in\left[2, \max _{i}\left\{2 N_{i}+2\right\}\right)$. Using the following lemma some precise estimates can be derived from Theorem 3.2.

Lemma 3.3 For any positive integers $m \geq 2, n \geq 0$ let $A_{k_{1}, \cdots, k_{m}}=1$ for all nonnegative integers $k_{1}, \cdots, k_{m}$ with $k_{1}+\cdots+k_{m}=n$. Then

$$
\sum_{k_{1}+\cdots+k_{m}=n} A_{k_{1}, \cdots, k_{m}}=C_{n+m-1}^{m-1} .
$$

Since $b_{2 q}\left(\mathbb{C} P^{n}\right)=1$ for $q=0,1, \cdots, n$, and $b_{q}\left(\mathbb{C} P^{n}\right)=0$ for other $q$,

$$
\operatorname{dim} R^{2 N}=\operatorname{dim} H^{2 N}(M)=\sum_{k_{1}+\cdots+k_{m}=N} b_{2 k_{1}}\left(\mathbb{C} P^{2 N_{1}}\right) \cdots b_{2 k_{m}}\left(\mathbb{C} P^{2 N_{m}}\right) .
$$

Let $N_{1}=\cdots=N_{m}=N$ and $2<d=2 l \leq 2 N+2$. Clearly, $A_{k_{1} \cdots k_{m}}:=$ $b_{2 k_{1}}\left(\mathbb{C} P^{2 N}\right) \cdots b_{2 k_{m}}\left(\mathbb{C} P^{2 N}\right)=1$ for any nonnegative integers $k_{1}, \cdots, k_{m}$ with 
$k_{1}+\cdots+k_{m}=N+1-l$. So the right side of (3.9) becomes

$$
\begin{aligned}
& \sum_{i=1}^{m} b_{2 N_{i}+2-d}(M)-\sum_{i=1}^{m} b_{2 N_{i}+2-d}\left(\mathbb{C} P^{2 N_{i}}\right) \\
= & m b_{2 N+2-2 l}(M)-m=m \operatorname{dim} H^{2 N+2-2 l}(M)-m \\
= & m \sum_{k_{1}+\cdots+k_{m}=N+1-l} b_{2 k_{1}}\left(\mathbb{C} P^{2 N}\right) \cdots b_{2 k_{m}}\left(\mathbb{C} P^{2 N}\right)-m \\
= & m \sum_{k_{1}+\cdots+k_{m}=N+1-l} A_{k_{1} \cdots k_{m}}-m=m C_{N+m-l}^{m-1}-m
\end{aligned}
$$

because of Lemma 3.3.

Similarly, for $d=2, N_{1}=\cdots=N_{m}=N$ the right side of (3.10) becomes

$$
\sum_{i=1}^{m} \operatorname{dim} R^{2 N_{i}}-m=m \operatorname{dim} R^{2 N}-m=m C_{N+m-1}^{m-1}-m .
$$

Hence we get the following (3.11).

Corollary 3.4 If $N_{1}=\cdots=N_{m}=N$ and $d=2 l \in[2,2 N+2]$ is even then

$$
\operatorname{rank}\left(\operatorname{coker} \beta_{d-1}\right) \geq m C_{N+m-l}^{m-1}-m \text {. }
$$

If $N_{1}<N_{2}=\cdots=N_{m}=N$ then

$$
\operatorname{rank}\left(\operatorname{coker} \beta_{1}\right) \geq C_{N_{1}+m-1}^{m-1}+(m-1) \sum_{k=0}^{N_{1}} C_{N+m-k-1}^{m-1}-m .
$$

For the proof of (3.12), since $N_{1}<N_{2}=\cdots=N_{m}=N$ we have

$$
\operatorname{dim} R^{2 N_{1}}=\sum_{k_{1}+\cdots+k_{m}=N_{1}} b_{2 k_{1}}\left(\mathbb{C} P^{2 N_{1}}\right) b_{2 k_{2}}\left(\mathbb{C} P^{2 N}\right) \cdots b_{2 k_{m}}\left(\mathbb{C} P^{2 N}\right)=C_{N_{1}+m-1}^{m-1}
$$

Note that $b_{2 k_{1}}\left(\mathbb{C} P^{2 N_{1}}\right)=0$ for $k_{1}>N_{1}$. Using Lemma 3.3 we deduce that

$$
\begin{aligned}
R^{2 N} & =\sum_{k_{1}+\cdots+k_{m}=N} b_{2 k_{1}}\left(\mathbb{C} P^{2 N_{1}}\right) b_{2 k_{2}}\left(\mathbb{C} P^{2 N}\right) \cdots b_{2 k_{m}}\left(\mathbb{C} P^{2 N}\right) \\
& =\sum_{k_{1}=0}^{N_{1}} \sum_{k_{2}+\cdots+k_{m}=N-k_{1}} b_{2 k_{2}}\left(\mathbb{C} P^{2 N}\right) \cdots b_{2 k_{m}}\left(\mathbb{C} P^{2 N}\right)=\sum_{k_{1}=0}^{N_{1}} C_{N+m-k_{1}-1}^{m-1} .
\end{aligned}
$$

Hence in this case the right side of (3.10) becomes

$$
\sum_{i=1}^{m} \operatorname{dim} R^{2 N_{i}}-m=C_{N_{1}+m-1}^{m-1}+(m-1) \sum_{k=0}^{N_{1}} C_{N+m-k-1}^{m-1}-m .
$$

In general, one can also give the estimate of $\operatorname{coker}\left(\beta_{d-1}\right)$ via(3.9) and (3.10) by tediously long and insipid computations. They are left to the interesting readers. 


\section{References}

[1] P. Seidel, On the group of symplectic automorphisms of $\mathbb{C} P^{m} \times \mathbb{C} P^{n}$. In: Northern California Symplectic Geometry Seminar, Amer. Math. Soc. Transl. Ser. 2, 196, Amer. Math. Soc., Providence, RI, 1999, 237-250.

[2] H. V. Lê, K. Ono, Parameterized Gromov-Witten invariants and topology of symplectomorphism groups. In: Groups of diffeomorphisms, Adv. Stud. Pure Math., 52, Math. Soc. Japan, Tokyo: 2008, 51-75.

[3] M. Gromov, Pseudo holomorphic curves in symplectic manifolds. Invent Math, 1985, 82: 307-347.

[4] J. McCleary, A user's guide to spectral sequences. Second edition. Cambridge Studies in Advanced Mathematics, vol. 58. Cambridge: Cambridge University Press, 2001.

\section{Received: April 15, 2013}

